\title{
Autour du concept d'expérience chez Benjamin
}

Expérience esthétique - expérience authentique

\section{Laurence Manesse}

\section{(2) OpenEdition \\ Journals}

Electronic version

URL: http://journals.openedition.org/appareil/1172

DOI: 10.4000/appareil. 1172

ISSN: 2101-0714

Publisher

MSH Paris Nord

\section{Electronic reference}

Laurence Manesse, "Autour du concept d'expérience chez Benjamin », Appareil [Online], Articles,

Online since 18 April 2013, connection on 30 July 2020. URL : http://journals.openedition.org/appareil/ 1172 ; DOI : https://doi.org/10.4000/appareil.1172

This text was automatically generated on 30 July 2020

\section{(c) (i) () $\Theta$}

Appareil est mis à disposition selon les termes de la Licence Creative Commons Attribution - Pas d'Utilisation Commerciale - Pas de Modification 4.0 International. 


\title{
Autour du concept d'expérience chez Benjamin
}

\author{
Expérience esthétique - expérience authentique
}

\author{
Laurence Manesse
}

\section{Que faut-il entendre chez Kant par expérience?}

Partons d'une citation :

"Que toute notre connaissance commence avec l'expérience, il n'y a là aucun doute ; car par quoi le pouvoir de connaître serait-il éveillé et mis en exercice, si cela ne se produisait pas par des objets qui frappent nos sens, et en partie produisent d'eux-mêmes des représentations, en partie mettent en mouvement notre activité intellectuelle pour comparer ces représentations, pour les lier ou les séparer, et élaborer ainsi la matière brute des impressions sensibles en une connaissance des objets, qui s'appelle expérience? Selon le temps, aucune connaissance ne précède donc en nous l'expérience, et toutes commencent avec elle.

Mais bien que toute notre connaissance commence avec l'expérience, elle ne résulte pas toute de l'expérience. Car il se pourrait bien que notre connaissance d'expérience elle-même soit un composé de ce que nous recevons par des impressions, et de ce que notre propre pouvoir de connaître (à l'occasion simplement des impressions sensibles), produit de lui-même, addition que nous ne distinguons de cette matière élémentaire, tant qu'un long exercice ne nous a pas rendus attentif à ce qui est ainsi ajouté et habiles à le séparer. $»^{1}$

Il aborde ici la question de l'expérience quant à son rôle dans la formation de la connaissance. Si l'expérience est bien reconnue comme fondement de la connaissance, elle apparaît néanmoins comme "matière" complexe qui ne fait pas seulement intervenir l'intuition, mais aussi une production de l'homme, c'est-à-dire le cadre, le concept. Dans notre expérience intervient naturellement la perception qui s'élabore à partir de concepts ou catégories. La perception elle-même est une construction élaborée par le sujet. Le moi, le sujet - en tant que conscience capable de se prendre elle-même comme objet de pensée - devient le « constructeur » de la nature, du monde, 
il en est le législateur en lui imposant ses lois. Ainsi la réalité sera composée de phénomènes, c'est-à-dire de représentations. Cette réalité est phénoménale, et non nouménale : la chose en soi est inconnaissable, nous dit Kant. Les limites sont ainsi posées.

3 Ainsi, l'expérience, en tant qu'intuition, observation, est chronologiquement (selon le temps) le premier moment de la connaissance, mais ces impressions sensibles ne suffisent pas, il ne peut y avoir de vraie connaissance sans concept! Ainsi si l'expérience en tant qu'intuition est le fondement de toute connaissance, elle est nécessaire mais non suffisante.

On est loin d'Aristote pour qui l'expérience commune, second degré du savoir, suffirait à une connaissance (sensation, perception, expérience, technique, la science, la sagesse) ; Pour Kant, toute connaissance fait déjà appel à la fois à l'intuition et à la faculté des concepts, c'est-à-dire l'entendement et ses catégories (règles permettant de constituer l'objectivité, modes de liaison les plus universels présupposés par les jugement; selon la quantité : unité, pluralité, totalité / selon la qualité : réalité, négation, limitation / selon la relation : substance, causalité, l'action réciproque / selon la modalité : la possibilité, l'existence, la nécessité) pour permettre la construction d'une réalité phénoménale.

5 L'expérience en général n'est donc pas donnée, elle est, grâce à des formes de connaissance a priori, élaboration d'un donné de nature sensible.

6 Donc l'expérience est le résultat d'une "collaboration" entre l'imagination et l'entendement: résultat de plusieurs synthèses: le monde nous touche par les sens (sensation), l'imagination opère une première synthèse, nous percevons le monde (conscience empirique); l'entendement opère une seconde synthèse, nous expérimentons le monde. Cette manière de le dire est un peu trop schématique pour être exacte, mais ceci nous permet je pense de mieux comprendre ce que Benjamin appelle une "expérience mécanique ", c'est-à-dire véritablement une construction du monde connaissable par le sujet à partir des sensations.

\section{La tâche de la philosophie à venir}

7 Pour Benjamin, en tout cas en 1917, « La tâche centrale de la philosophie qui vient est d'élever à la connaissance, en les rapportant au système de Kant, les intuitions les plus profondes qu'elle puise dans son époque et dans le pressentiment d'un grand avenir. $»^{2}$

8 Nous voici propulsé dans l'histoire... l'histoire des hommes, leur époque et leur destination. On peut dire d'une certaine manière dans deux dimensions de la temporalité. Le programme va donc consister à faire de ces intuitions (dans ma lecture je suppose ici «intuitions intellectuelles») et pressentiments, de véritables connaissances.

9 Or, que nous dit Kant, des pressentiments? Il a établi trois degrés dans l'acte de tenir pour vrai : le savoir, la croyance et l'opinion jusqu'à parvenir à l'ignorance. Il y ajoute un nouveau degré : le pressentiment du suprasensible.

«Or, qu'il y ait ici un certain tact mystique, un saut des concepts à l'impensable, un pouvoir de s'emparer de ce qu'aucun concept n'atteint, une attente de mystères, ou plutôt la promesse réitérée qu'on y accèdera, mais en fait, à la vérité une fâcheuse disposition des cerveaux à l'exaltation, c'est l'évidence. Car le pressentiment est une attente obscure et contient l'espoir d'une solution qui n'est toutefois possible 
que par concepts, dans les tâches de la raison, alors que ces questions sont transcendantes et ne peuvent conduire à aucune véritable connaissance de l'objet, il faut nécessairement qu'elles en promettent un succédané, une communication surnaturelle (une illumination mystique): ce qui est alors la mort de toute philosophie $»^{3}$

10 Alors pourquoi vouloir se rapporter au système kantien? Parce que Kant est un des rares philosophes « à ne pas se soucier immédiatement de l'étendue et de la profondeur de la connaissance, mais surtout, et en premier lieu, de sa justification ». Justification et profondeur sont liées dans la philosophie.

11 Kant ajoutait d'ailleurs sur le pressentiment qu'il est une vue de l'avenir, il « suggère en quelque sorte un sens caché pour ce qui n'est pas encore présent ", un tel pressentiment est une chimère, car «comment avoir la sensation de ce qui n'est pas encore? " Les pressentiments sont tout au plus des jugements issus de concepts obscurs, mais l'on peut « développer les concepts qui y mènent. " $^{4}$ On peut presque affirmer, que Kant lui-même, déduisant de l'enthousiasme du peuple allemand spectateur de la Révolution française, un progrès irréversible de l'humanité vers le mieux, vers la liberté, a opéré ce développement. Il écrit en effet dans Le conflit des facultés :

«Or, je prétends pouvoir prédire au genre humain - et cela même sans esprit prophétique - d'après les aspects et les signes précurseurs qui sont visibles de nos jours, qu'il atteindra cette fin et en outre, en même temps, que son progrès vers le mieux ne sera plus susceptible, désormais, d'un renversement total. $»^{5}$

Il y a donc cependant un mais que Benjamin pointe clairement :

«Mais une philosophie véritablement consciente de son temps et de l'éternité, cherchant à se rattacher à Kant, se heurtera principalement à l'obstacle suivant : la réalité dont ce philosophe voulait fonder la connaissance, et avec laquelle il voulait fonder la connaissance sur la certitude et la vérité, est une réalité de rang inférieur, peut-être du dernier rang. "

14 Cette réalité est celle des Lumières qui induit une certaine vision du monde inscrite dans une époque. «Que Kant ait pu justement entreprendre son œuvre immense sous le signe des Lumières signifie qu'il est parti d'une expérience réduite en quelque sorte au point zéro, à un minimum de signification » parce que marquée par le modèle mathématique.

Il s'explique plus loin :

«Pour suggérer ce qui fait la pauvreté et la médiocrité de l'expérience propre à cette époque, à quoi tient son étonnante inconsistance métaphysique, il suffit de mesurer l'influence restrictive que ce concept inférieur d'expérience a exercée sur la pensée kantienne elle-même. Nous voulons naturellement parler de cette cécité religieuse et historique que l'on a souvent reproché aux Lumières, sans reconnaître en quel sens ces caractères sont imputables à l'ensemble des Temps modernes. ${ }^{6}$

Voici le projet se préciser :

«La philosophie que nous attendons devra justement s'attacher à découvrir et à mettre clairement en lumière dans le système kantien une certaine typologie capable de rendre justice à un mode d'expérience supérieur. [...] Il s'agit donc, sur la base de la typologie kantienne, de poser les prolégomènes d'une métaphysique future et en même temps d'ouvrir une perspective sur cette métaphysique future, cette expérience supérieure. » 
17 Cependant, il faudrait repartir, pense-t-il alors, de la question de la connaissance, mieux du rapport établit par Kant entre expérience et connaissance, pour ouvrir à une métaphysique future.

«La théorie kantienne de la connaissance ne débouche pas sur le domaine de la métaphysique, parce qu'elle porte en elle-même des éléments primitifs d'une métaphysique inféconde (métaphysique spéculative, inféconde), exclusive de toute autre. Dans la théorie de la connaissance, toute métaphysique est un germe morbide qui trouve son expression dans l'isolement de la connaissance par rapport au domaine de l'expérience envisagée dans sa liberté, sa profondeur pleines et entières. "

18 Il faut donc repartir de l'expérience supérieure (dans sa liberté, sa profondeur pleines et entières) pour ouvrir aux vérités métaphysiques, revoir cette théorie de la connaissance qui n'a pas su pointer le lieu logique de la recherche métaphysique, qui serait donc à trouver ou au moins à chercher. La première difficulté à surmonter consisterait à dépasser la relation sujet/objet. La seconde serait de reconsidérer la relation connaissance/expérience en lien avec la conscience empirique de l'homme. Les deux aspects sont, on le sent bien, étroitement liés. Pas de relations sujet/objet sans conscience de soi, éprouvée, expérimentée. Pour le dire autrement, sans cette capacité à se distinguer du monde: je me pense comme sujet dans le monde, je suis cette conscience consciente d'elle-même dans le monde qui est autre que moi.

Il s'agirait d'« éliminer la nature subjective de la conscience connaissante » précise Benjamin. Cette difficulté vient de ce que la conscience connaissante se forme par analogie avec la conscience empirique (qui a bien des objets se tenant là, devant elle).

Benjamin ajoute : «...on ne peut douter du rôle primordial que joue dans le concept kantien de connaissance l'idée, fût-elle sublimée, d'un moi individuel, à la fois corporel et intellectuel, qui, au moyen des sens, reçoit des sensations à partir desquelles il constitue (on pourrait dire il construit) ses représentation. $»^{7}$

21 Or cette conception relève de la mythologie, précise Benjamin, et elle n'a aucune valeur quant à son contenu de vérité. Il le montre en faisant référence à d'autres mythologies de la connaissance rencontrées chez les peuples primitifs au stade pré-animiste qui s'identifient aux plantes et aux animaux sacrés, dont ils prennent le nom ; ou encore à certaines pathologies où le sujet s'identifie à des objets qu'il perçoit (la distinction sujet/objet disparait); ou encore les médiums prétendant ressentir les perceptions d'autrui comme les leurs.

22 Penser la distinction sujet/objet comme nécessaire revêt la même valeur de vérité, c'est-à-dire toute relative, voire douteuse. Aussi Benjamin affirme: "L'homme qui connaît, la conscience empirique connaissante, est une variété de la conscience délirante. » Il n'existerait donc que des différences de degré entre diverses espèces de conscience connaissante. Or, s'il y a des différences de valeur entre ces différents degrés de conscience connaissante, le critère ne peut en être la justesse des connaissances puisqu'elle n'intervient jamais au niveau empirique, c'est-à-dire psychologique.

23 Ainsi : «Établir le vrai critère de la différence de valeur entre les diverses espèces de conscience, telle sera l'une des plus hautes tâches de la philosophie qui vient. Aux espèces de la conscience empirique correspondent autant d'espèces d'expériences, lesquelles, du point de vue de leur relation à la vérité de la conscience empirique, n'ont pas plus de valeur que l'imagination ou l'hallucination. » pourquoi ? « parce qu'aucune 
relation objective n'est possible entre la conscience empirique et le concept objectif d'expérience. »

On voit bien ici que ce qui est essentiellement reproché à Kant est d'écarter d'un revers de plume tout type d'expérience autre que celle possible dans un rapport normé au monde. Normé ou réglé par la conscience de soi.

\section{3. À la recherche de l'expérience absolue}

La notion d'expérience inclue pour Benjamin, nous rapporte Scholem ${ }^{8}$, notamment les rapports spirituels et psychologiques qui se créent entre l'homme et l'univers dans les domaines où la connaissance n'a pas encore pénétré. Scholem lui fit un jour remarquer à l'occasion d'une de leurs conversions autour de cette philosophie à venir: " ... logiquement, il faut donc inclure les disciplines mantiques dans cette notion d'expérience» (divination). Benjamin lui aurait répondu: "Une philosophie qui n'inclut pas, et ne peut pas expliquer, la possibilité de lire l'avenir dans le marc de café n'est pas une philosophie authentique. »

Il ajoute : «Peut-être ce genre de divination est-il condamnable de certains points de vue, et notamment du point de vue du judaïsme, mais il faut en admettre la possibilité à partir du contexte naturel des choses." Il faut peut être penser ici à la vision rencontrée dans l'antiquité d'un ordre cosmique, universel.

On peut dès lors comprendre l'intérêt de Benjamin pour les expériences liées à la consommation de haschisch par exemple, sa lecture Les paradis artificiels de Baudelaire, ou pour l'hallucination comme mode de l'expérience humaine qui ne serait pas à rapprocher de l'illusion.

Toujours selon Scholem, Benjamin ne s'intéressait pas vraiment à la question du positivisme rationaliste ; ce qu'il cherchait surtout c'était «l'expérience absolue ».

Scholem raconte encore : «Il me lut un jour une assez longue note qu'il avait rédigée à propos du rêve et de la voyance; dans ce texte, il essayait également de formuler les lois qui gouvernaient le monde des fantasmes pré-mytiques. Il distinguait deux ères historiques, celle des fantasmes et celle des démons, qui, selon lui avaient précédé l'ère de la Révélation" ou l'ère messianique. "Le contenu véritable du mythe, selon Benjamin, était l'immense révolution qui, s'opposant aux fantasmes, avait mis fin à l'ère correspondante. Dès cette époque, il était préoccupé par le problème de la perception. [...] Dans ces réflexions se trouvaient déjà en germe les idées qu'il allait exposer bien des années plus tard dans son écrit « Théorie du semblable ».

Ou encore il affirmait que la naissance des constellations en tant que configurations de la surface céleste constituait le début de la lecture et de l'écriture, et qu'elle coïncidait avec la naissance de l'ère mythique; les constellations avaient été pour le monde mythique ce qu'allait être plus tard la Révélation de l'Écriture sainte.

«Le spectre des états intermédiaires existant entre le rêve et l'état de veille le

fascinait tout autant que le rêve lui-même. »

31 Scholem rapporte qu'il avait élaboré une loi d'interprétation des rêves, ce qui marque une époque de ses préoccupations, où il consacrait du temps à analyser ses propres rêves.

Ceci pour insister sur la dimension multiple de l'expérience pour Benjamin, et son refus d'y introduire une classification fondée sur une quelconque valeur de vérité. Ce que 
j'expérimente je l'expérimente effectivement. L'expérience, qu'elle soit celle de l'hallucination, du rêve, de la réflexion, de la contemplation, de la rêverie, voire peut être même du délire, est source de connaissance parce qu'elle a une réalité "pour moi». Mais cette manière de l'exprimer reste problématique, puisqu'il s'agit précisément de suspendre ce moi dans notre rapport au monde, alors peut être une réalité qui me dépasse, ou qui « me convoque » ? Ou encore une réalité qui porte en elle une certaine valeur de vérité ne serait-ce peut être que par sa seule possibilité.

Bref, revenons à cette expérience authentique qui repose pour Benjamin, sur la pure conscience transcendantale définie au plan de la théorie de la connaissance. Le problème va être de pouvoir maintenir le terme de conscience en l'absence du sujet.

Il n'en demeure pas moins que cette notion de "pure conscience transcendantale » est bien différente de celle de " conscience empirique ». Nous renvoie-t-elle au Je-sans-moi du sentiment du beau ? Il semblerait que la subjectivité y soit encore trop présente. Au sentiment du sublime peut-être, si on peut y supposer une pensée sans sujet, une pensée pure pour une conscience transcendantale ? Cette question serait à reprendre.

Il s'agit donc pour Benjamin de déployer une théorie de la connaissance qui permettrait de dépasser complètement cette dichotomie sujet/objet, autrement dit : « de découvrir la sphère autonome et originaire de la connaissance où ce concept ne définit plus d'aucune manière la relation entre deux entités métaphysiques. » (p. 187) ce qui ne dispense pas de rester en conformité avec la relation découverte par Kant entre connaissance et expérience, à savoir que l'expérience est la condition de la connaissance. Seulement la question de la conscience empirique serait dépassée. C'est là que se réinstallerait la possibilité logique de la métaphysique.

Nous laisserons là le texte de Benjamin pour nous permettre maintenant de tenter des rapprochements entre la question de l'expérience esthétique comme expérience authentique.

\section{De l'expérience authentique à l'expérience esthétique}

37 Il semble toutefois que cette notion d'expérience authentique peut être précisée à partir de ce texte, et il devient ici absolument évident que la question de l'aura et de sa disparition est bien celle d'un cas de l'expérience authentique.

«Qu'est-ce au juste que l'aura? Une trame singulière d'espace et de temps: l'unique apparition d'un lointain, si proche soit-il. Un jour d'été en plein midi, suivre du regard la ligne d'une chaîne de montagnes à l'horizon ou d'une branche qui jette son ombre sur le spectateur, jusqu'à ce que l'instant ou l'heure ait part à leur manifestation - c'est respirer l'aura de ces montagnes, de cette branche. ${ }^{9}$

Ici l'expérience serait celle d'une respiration, une respiration dans laquelle semble se mêler toute dimension temporelle et spatiale (passé présent futur - ou encore immédiateté et éternité - proximité et éloignement); pour le dire autrement une expérience dans la respiration d'un ici maintenant, aux saveurs d'un là-bas hier, relevé par un de tout temps en tout lieu, peut être... Une expérience qui ne relèverait pas de la construction du sujet mais de cette cohabitation spontanée du corps (respiration) de la pensée (espace et temps) et du monde dans l'unicité de l'apparition de l'aura. 
Nous n'attribuons pas une qualité à un objet mais nous ne faisons rien d'autre que de mettre un mot sur une certaine disposition de notre esprit en tant que mise en rapport toute particulière de nos facultés, à l'occasion d'un objet. Si le « c'est beau » désigne un libre jeu harmonieux entre imagination et entendement, le «c'est sublime » désigne bien plutôt un désaccord, une disharmonie entre imagination et raison qui cependant, et c'est là le moment sublime, engendre l'illimitation de l'imagination tirée vers le suprasensible par la raison.

« [le sentiment du sublime] est un plaisir qui ne surgit que de manière indirecte, c'est-à-dire qu'il est produit par le sentiment d'un soudain blocage des forces vitales, suivi aussitôt d'un épanchement d'autant plus puissant de celles-ci; ; en tant qu'émotion, le sentiment du sublime ne semble pas un jeu mais une activité sérieuse de l'imagination. $»^{11}$

46 
qu'elle ne parvient pas à résister à la puissance de la nature - sublime dynamique -, lui donne ce sérieux dans le sublime.

Ici un double mouvement, d'abord l'anéantissement de l'imagination dans son effort de présentation puis son soulèvement, son emportement dans l'illimitation du suprasensible. Il s'agit bien d'un mouvement dynamique, du déploiement des forces vitales, d'une puissance de l'esprit. La raison conçoit l'absolu, à l'imagination de le lui présenter. Et la raison, comme le dit Lyotard, a raison d'exiger d'elle une telle présentation, même si ses efforts pour y parvenir resteront vains, puisque la raison est ici pratique et que l'Idée à présenter est la causalité inconditionnée, la liberté, qui requiert constitutivement son effectuation présente mais qui, aussi, constitue la destination suprême de l'esprit, on peut dire de l'homme.

La raison est un pouvoir de concevoir des objets de pensée, objets intelligibles, c'est-àdire des Idées dont elle est la source et pour lesquelles il n'y a pas de présentation possible dans l'expérience. Il s'agit d'absolus ou d'illimités alors que la " compréhension » qui permet la présentation des sensations en une unité a une limite qui consiste en une mise en forme, et que la forme est une limitation. Ainsi,

«... le sublime pourra être trouvé aussi en un objet informe, pour autant que l' illimité sera représenté en lui ou grâce à lui et que néanmoins s'y ajoutera par la pensée la notion de sa totalité. »

C'est l'illimité qui doit être présenté, c'est-à-dire sollicité dans l'esprit, l'illimité dans sa totalité, et on sent le paradoxe, ce que l'imagination ne peut parvenir à faire ; c'est là son échec, jusqu'à ce qu'elle parvienne à sentir, à rendre sensible, le suprasensible, qui sera son emportement.

Kant, dans ses «Remarques générales sur l'exposition des jugements esthétiques réfléchissants » écrit :

«On ne doit pas se soucier de ce que le sentiment du sublime soit perdu par un mode de présentation aussi abstrait, tout à fait négatif eu égard à la sensibilité ; en effet, bien qu'elle ne trouve rien au-delà de la sensibilité à quoi se rattacher, l'imagination se sent néanmoins illimitée en raison même de l'abolition des barrières de la sensibilité ; et cette abstraction est donc une présentation de l'infini qui, précisément pour cette raison, ne peut jamais être autre chose qu'une présentation négative, laquelle cependant élargit l'âme. $\aleph^{12}$

51 Il est évident que dans le sentiment du sublime, quelque chose arrive à l'imagination, elle s'illimite dans la présentation à l'esprit du suprasensible et cette illimitation est le résultat du dépassement du sensible par la relève assurée par la raison.

Ses bornes disparaissent, nous dit Kant. Alors, quelles sont ces bornes de l'imagination qui se voient volatilisées dans le sublime? L'imagination n'est plus bornée par le sensible, et ce qui se soulève c'est la liberté de l'imagination. Son activité reproductive se suspend, il ne s'agit plus de liaison entre les représentations suivant les lois d'association, il ne s'agit plus de représenter, puisqu'un grand $\mathrm{X}$ transcendantal se présente hors représentation. Son activité productive se suspend, il n'est plus question d'appliquer dans la liaison les lois de l'entendement, de toutes façons l'entendement est ici hors jeu.

Il reste une intuition sensible, là encore une occasion, qui sera le détonateur propulsant l'imagination dans la sphère du suprasensible où il ne saurait plus être question de représentation mais de présentation, une présentation qui ne fera pas représentation. 
54 L'imagination perçoit bien quelque chose qu'elle ne constituera pas en objet, en tout cas pas en objet de l'expérience, parce que, s'il y a expérience, elle ne sera que de la pensée. Et on touche peut être ici le point d'articulation avec la question de l'expérience authentique de Benjamin. Reste à savoir si nous dépassons ici cette fameuse dichotomie sujet/objet...

De là, le sublime est l'expérience d'une non-expérience dans le sensible, mais aussi l'expérience du suprasensible, de la pensée pure ou transcendantale; il est ce passage du sensible au suprasensible à l'occasion d'un objet.

En ce sens encore, nous pouvons parler, avec le sentiment ou jugement esthétique, d'une expérience interne, toujours liée à la présentation d'un objet comme présence, mais encore allant au-delà de l'expérience du sujet pour lui-même. En effet, dans le beau, le sujet s'expérimente comme je-sans-moi, comme sujet universel à qui se signale dans le beau, cette part d'humanité qui est en lui, qui fait son essence même en tant que condition de possibilité de toute pensée (ou connaissance) à venir ; dans le sublime, le sujet s'expérimente en tant que partie d'un tout comme humanité hors de lui en tant que projet. Autrement dit, il s'expérimente dans une tout autre dimension que la conscience de soi. Cette expérience interne, que Kant pense comme «conscience immédiate et évidente» dans ses Principes de la théologie naturelle I p. 230, présuppose toujours d'abord l'expérience externe, ou mieux ici, que quelque chose se présente à l'esprit dans le sensible, quelque chose qui aura perdu finalement son statut d'objet. Cette conscience est liée à «la conscience d'un rapport à quelque chose hors de moi. » Kant, Critique de la raison pure, op.cit., t. I, p. 955-957. Il serait intéressant ici de s'attarder sur la nature de ce rapport puisqu'il n'est plus de même nature que le rapport sujet/ objet, qui est bien ce que Benjamin propose de dépasser.

Le propos ou le rôle de l'imagination n'est plus la représentation empirique des phénomènes dans l'association et la reproduction, puisque quand elle tente de maintenir ce rôle, elle s'essouffle et s'épuise sans y parvenir. C'est ce premier moment de "soumission » à la raison qui fait sa souffrance, par cette violence même de la raison, pour s'illimiter dans le suprasensible, puisque libérée de ses contraintes liées à la représentation ou présentation sensible.

Ce sentiment s'éveille, comme le beau, à l'occasion de quelque chose dont nous ne saurons finalement pas grand chose si ce n'est qu'il demeure sous le signe du paradoxe : «[...] pour autant que l'illimité sera représenté en lui [...] s'y ajoutera par la pensée la notion de sa totalité " Il conviendra à la présentation d'un concept indéterminé de la raison. Sentiment d'un arrêt des forces vitales immédiatement suivi d'un épanchement de celles-ci : plaisir négatif. Il comprend admiration et respect :

«La nuit est sublime, le jour est beau. Ceux qui possèdent le sentiment du sublime sont portés aux sentiments élevés... $»^{13}$

C'est quand la nature se dérègle, se déchaîne qu'elle est la plus propice à susciter le sublime en nous. Il s'agit de rendre sensible en nous-mêmes une finalité tout à fait indépendante de la nature. N'entendons nous pas ici comme un écho au " pressentiment d'un grand avenir » ? L'esprit est appelé à se détacher de la sensibilité, à la transcender, et à se consacrer aux Idées, au suprasensible. Le sublime est l'expérience de la limite de la forme, l'expérience aussi des limites de l'esprit, l'expérience d'une pensée hors des contraintes des catégories de l'entendement mais aussi des contraintes de l'espace et du temps, des contraintes de la connaissance au sens de la première critique. N'y aurait-il pas cependant quelque chose comme une 
connaissance à y trouver, à la manière d'une « révélation » peut-être, d'une expérience comme révélation d'une conscience transcendantale?

\section{NOTES}

1. Kant, "Critique de la raison pure", Euvres philosophiques I, Gallimard, Bibliothèque de la pléiade, 1980, p. 757.

2. Walter Benjamin, "Sur le programme de la philosophie qui vient», Euvres I, Folio essais, p. 179.

3. Kant, "Sur un ton supérieur nouvellement pris en philosophie ", Euvres philosophiques III, Gallimard, Bibliothèque de la pléiade, 1980, p. 404-406.

4. Kant, « Anthropologie du point de vue pragmatique », Euvres philosophiques III, p. 1004.

5. Ibid., « Le conflit des facultés », p. 899-900.

6. Benjamin, op.cit., p. 182.

7. Benjamin, op.cit., p. 185.

8. Gershom Scholem, Walter Benjamin, histoire d'une amitié, Calmann-Lévy, 1981, p. 75.

9. Walter Benjamin, « Petite histoire de la photographie », Euvre II, p. 311.

10. Antelme R., L'Espèce Humaine - Ed. Tel 1997, p. 232.

11. Kant, Critique de la faculté de juger, op. cit., t. II, p. 1010.

12. Ibid.

13. Kant, Observation sur le sentiment du beau et du sublime, op.cit., t. I, p. 453.

\section{ABSTRACTS}

Notre attention a été retenue par un texte programmatique rédigé en 1917-1918 par Benjamin, texte de jeunesse, sous le titre Sur le programme de la philosophie qui vient, dont l'enjeu central serait une théorie de l'expérience établie à partir de la critique du concept kantien.

Ce programme, Benjamin l'enracine dans la reprise de la question de l'expérience, et donc de la connaissance chez Kant. Le reproche qu'il oppose à Kant est d'être parti de ce qu'on pourrait appeler, le degré zéro de l'expérience.

Nous nous proposons donc de commencer par comprendre ce qu'est l'expérience chez Kant et en quoi elle pourrait être le degré zéro de l'expérience. Nous reviendrons ensuite sur ce qu'est chez Benjamin l'expérience authentique pour établir un rapprochement, si rapprochement il peut y avoir entre expérience authentique et expérience esthétique (selon Kant). Enfin se demander si l'expérience du sublime peut être pensée comme un cas de l'expérience authentique. 